\title{
Políticas de Mobilidade Urbana: Identificação de Critérios de Avaliação das Políticas e Ações de Ciclomobilidade
}

Urban Mobility Policies: identification of evaluation criteria of cyclomobility policies and actions.

Wellington Luiz de Azevedo*, Flavia Nico Vasconcelos** e Giovanilton André Carreta Ferreira ${ }^{\star \star \star}$

*Profissional em arquitetura e urbanismo graduado pela Universidade Vila Velha em 2015. Atualmente cursando Mestrado em Arquitetura e Cidade pela Universidade Vila Velha - ES, integrante do grupo de pesquisa Dignidade Urbana (UVV, 20172018) participando nos seguintes temas: Planejamento e Projeto de Espaços Livres de Uso Público, Aspectos Físico-Ambientais e Sociais do Planejamento Urbano e Regional, Vitalidade Urbana.

${ }^{* \star}$ Experiência internacional como visiting scholar: no curso Engenharia do Território do Instituto Superior Técnico de Lisboa (2008) e no Urban Studies Program, da Simon Fraser University, Vancouver (2014). Professora do Curso de Graduação em Relações
Internacionais e professora do Mestrado Sociologia Política na Universidade Vila VeIha. Pesquisa sobre cidades e portos, principalmente nos temas globalização, cidades portuárias e cidades inteligentes.

${ }^{* *}$ Conselheiro suplente do Conselho Arquitetura e Urbanismo do Espírito Santo. Líder do Grupo Pesquisa Cidade e Políticas Urbanas (UVV UFES/FAACZ), membro do Grupo Pesquisa Grandes Projetos de Desenvolvimento Urbano (UFF). Tem experiência na área de Arquitetura e Urbanismo, com ênfase em Planejamento Urbano e Regional e na elaboração de Planos, Programas e Projetos de Ordenamento Territorial e de gestão da cidade.

\section{Resumo}

A valorização dos modos de transporte não motorizados tem se mostrado evidente nas esferas do governo federal, estadual e municipal, e tem impulsionado a discussão sobre a mobilidade cicloviária, onde em meio ao caos na mobilidade urbana ocasionado pelo modelo de circulação baseado no automóvel, fez com que os governos incluíssem de forma significativa políticas públicas, legislações e ações voltadas a mobilidade cicloviária com novas alternativas para problemas associados à mobilidade urbana. Contudo no Brasil há uma lacuna quanto à necessidade do estabelecimento de critérios para avaliar a evolução da implantação das políticas e ações de mobilidade cicloviária nos municípios. Dessa forma o estudo propõe com base na legislação brasileira e experiências nacionais e internacionais levantar critérios que permitam avaliar as políticas ações de ciclomobilidade implantadas, de forma a ser um instrumento aplicável nos municípios.

Palavras-chave: Mobilidade Cicloviária. Critérios de Avaliação de Mobilidade. Bicicletas. Mobilidade Urbana. Gestão Urbana de Mobilidade.

\section{Abstract}

The valuation of non-motorized modes of transport has been evident in the federal, state and municipa government spheres, and has stimulated the discussion on cycling mobility, where, amidst the chaos in urban mobility caused by the automobilebased circulation model, governments to include in a significant way public policies, legislation and actions aimed at cycling mobility with new alternatives to problems associated with urban mobility. However, there is a gap in Brazil regarding the need to establish criteria to evaluate the evolution of the implementation of policies and actions of cycling mobility in municipalities. In this way, the study proposes, based on Brazilian legislation and national and international experiences, to establish criteria that allow the evaluation of the policies and actions of cyclomobilization implemented, in order to be an applicable instrument in the municipalities.

Keywords: Keywords: Cycle mobility. Criteria for Mobility Assessment. Bicycles. Urban mobility. Urban Mobility Management. 


\section{Introdução}

Em consonância com um projeto maior em que a Política Nacional de Mobilidade Urbana (PNMU) orienta a necessidade de estabelecer critérios para avaliar a evolução das políticas de mobilidade cicloviária nas cidades, este artigo é decorrente de dissertação de mestrado e explora os instrumentos de política pública, normativas de mobilidade urbana de ciclomobilidade, bem como os principais documentos de referência em uso que abordam a temática de critérios de avaliação das políticas e ações de ciclomobilidade, com propósito de apresentar um instrumento com critérios para avaliar a evolução das políticas de mobilidade nas cidades.

Para tanto, iniciamos este artigo discutindo a mobilidade urbana e seus desafios nas cidades contemporâneas. Por este caminho, apontamos a ciclomobilidade como uma alternativa sustentável e que meramente a implantação das políticas das infraestruturas cicloviárias não trazem resultados satisfatórios, carecendo de critérios para avaliação. Em sequência iremos abordar o marco legal - O que diz a legislação sobre critérios de avaliação das políticas e ações de mobilidade cicloviária nos municípios. Prosseguiremos apresentando - Uma proposta de critérios de avaliação das políticas públicas locais do modal cicloviário, onde abordaremos algumas das referências de critérios de avaliação da legislação em ambientes nacionais e internacionais, como possibilidade de contribuição na gestão das ações cicloviárias nos municípios, sendo elas: - $A$ orientação legal brasileira: Caderno Bicicleta Brasil, - A experiência brasileira: o relatório de ITDP sobre São Paulo e - The Copenhagenize Index: a classificação no ranking mundial, obtendo como resultado um instrumento de avaliação de política cicloviária nos municípios. Finalizaremos o artigo com as Considerações Finais. 


\section{Ciclomobilidade: Importância dos Critérios de Avaliação}

A ocupação do espaço público pelos automóveis está associada às mudanças na dinâmica urbana das cidades contemporâneas e suas múltiplas possibilidades de infraestruturas e de equipamentos do meio urbano (cultura, comércio, formação, serviços, atividades sociais e políticas). A partir das primeiras décadas do século $X X$, acreditou-se que o automóvel garantia a necessidade de acessibilidade a essas possibilidades, preservando, assim, o interesse geral.

A cidade moderna elegeu o automóvel como principal meio de locomoção em detrimento do transporte público e do transporte não motorizado. Assim, passou a lidar com várias consequências, como lentidão de deslocamentos, poluição e redução do número de usuários no sistema de transporte público (ANTP, 1997). Mas, os problemas associados a esse modelo de circulação não se limitam à cidade, seus impactos também são sentidos globalmente: alterações climáticas e aquecimento global, problemas de saúde, dentre outros.

A ideia de mobilidade urbana é tradicionalmente vinculada ao transporte. Atualmente, o conceito de mobilidade urbana tem interpretação ampliada para considerar a "condição em que se realizam os deslocamentos de pessoas e cargas no espaço urbano", incluindo - e priorizando-os modos de transporte coletivos e não motorizados (BRASIL, 2012). Em sua acepção contemporânea, a mobilidade urbana está relacionada com a qualidade de vida urbana e deve estar articulada ao plano de desenvolvimento da cidade e à democratização dos espaços públicos. Ou seja, prioriza as pessoas no lugar dos automóveis.

Seguindo a tendência internacional de promoção de medidas e políticas sustentáveis, alguns estudos têm apresentado a bicicleta como um meio de transporte viável, capaz de interagir eficientemente com todas as outras formas de mobilidade urbana, além de proporcionar a melhoria do meio ambiente e ajudar a promover a inclusão social (IEMA, 2010). Logo, as bicicletas e o modal cicloviário aparecem como uma solução sustentável de mobilidade urbana.

A busca por novas alternativas para os problemas associados à mobilidade urbana, tem incluído por parte dos governos a ciclomobilidade de forma significativa nas políticas, nos planos, na implantação de infraestruturas e ações relacionadas a mobilidade urbana, isto é, que buscam alternativas de transporte sustentável de forma geral. Contudo somente ações de elaboração de políticas e implantação de uma ciclovia pode não 
trazer resultados satisfatórios em termos de meIhoria na mobilidade e a evolução na implantação das políticas de mobilidade, sendo um fator relevante para seu sucesso o uso de mecanismos de avaliação sistemáticos.

Nesse sentido a Política Nacional de Mobilidade Urbana (PNMU) em seu Capítulo V orienta que as diretrizes para o Planejamento e Gestão dos Sistemas de Mobilidade Urbana, deverão contemplar a formulação e implantação dos mecanismos de avaliação e monitoramento sistemáticos e permanentes por indicadores preestabelecidos com o objetivo de garantir a consecução das metas de universalização e de qualidade (BRASIL, 2012).

Com o propósito de elaborar mecanismos de avaliação e monitoramento sistemático o $\mathrm{MC}$ no ano de 2016 elabora o relatório de "Indicadores de Efetividade da Política Nacional de Mobilidade Urbana", para definir os indicadores para avaliar e monitorar a efetividade da PNMU, contudo o relatório foi apresentado de forma parcial, com a lista de indicadores de curto prazo, e futuramente o relatório será apresentado de forma completa com indicadores de médio e longo prazo. Podemos observar ainda que o relatório faz uma consideração que não avalia o modal cicloviário, sendo: "Entende-se que esta lista ainda possui lacunas no que se refere aos indicadores para avaliar as condições de mobilidade e nos deslocamentos a pé e por bicicletas (...)". O que demonstra necessidade de criar critérios para avaliar a implantação das políticas de ciclomobilidade (MINISTÉRIO DAS CIDADES, 2016).

Assim, a realização deste trabalho justifica-se na medida em que existe uma lacuna, espaço pouco explorado, quanto à necessidade do estabelecimento de critérios para avaliar a evolução das políticas de mobilidade cicloviária nas cidades, que estabelecidos podem ser um relevante instrumento que proporcione ao ciclista avaliar o desempenho da gestão pública e ao gestor público conhecer o resultado do serviço que administra, bem como tomar decisões e direcionar/controlar os recursos e atingir objetivos da organização de forma eficiente e eficaz.

Para melhor compreensão dos termos, Casley e Kumar (1987) entendem que monitorar é uma avaliação contínua, tanto do funcionamento das atividades de um projeto no contexto de seus programas de implementação quanto no uso dos insumos do projeto pelas populações envolvidas, e no contexto das expectativas planejadas. Já avaliar é uma ação periódica, que está relacionada à importância, desempenho, eficiência e expectativa do projeto no âmbito dos objetivos iniciais estabelecidos. Em geral, envolve comparações que podem requerer informação externa ao projeto no tempo, área e população.

Segundo Garcia (2001) a avaliação é uma operação na qual são julgados valores originários de 
uma iniciativa organizacional, tendo como premissa padrão comparativo ou quadro de referências estabelecido a priori; ou pode ser considerada, de outra forma como a operação de verificar a presença ou a quantidade de um valor desejado nos resultados da referida operação para conhecê-la, tendo como princípio um quadro de critérios ou de referências de admissibilidade pretendidos.

Assim podemos entender que avaliação e o monitoramento na prática são resultados, efeitos ou impacto dos programas que se implementam, os termos são utilizados de maneira conjunta e sinônima, mas diferem-se. A diferença é que o monitoramento é uma avaliação contínua e a avaliação é conduzida periodicamente.

Logo, a questão é levantar os critérios para avaliar a implantação das políticas de ciclomobilidade, com base na legislação brasileira e experiências nacionais e internacionais.

Dessa forma o objetivo é apresentar critérios que permitam avaliar as políticas e ações de mobilidade cicloviária implantadas nos municípios. A metodologia utilizada para alcançar o objetivo proposto foi de pesquisa bibliográfica com o propósito de aprofundar os conhecimentos concernentes à mobilidade cicloviária. A pesquisa contemplou literaturas consagradas (livros), as produções acadêmicas (teses e dissertações), artigos científicos (periódicos e eventos) e ins- trumentos de política pública e as normativas de mobilidade urbana que tangenciam a ciclomobilidade como o "Planejamento Cicloviário - Uma Política para as Bicicletas", da Empresa Brasileira de Planejamento de Transporte (GEIPOT, 2001), Estatuto da Cidade ou Lei 10.257/01, os documentos Caderno de Referência para Elaboração de Plano de Mobilidade Urbana (MINISTÉRIO DAS CIDADES, 2007a) e o Caderno de Referência para Elaboração de Plano de Mobilidade por Bicicleta nas Cidades (MINISTÉRIO DAS CIDADES, 2007b), e a Política Nacional de Mobilidade Urbana (PNMU) ou Lei 12.587/2012.

Para o propósito de elencar os critérios de avaliação das políticas e ações do modal cicloviário, utilizaremos três documentos de referência: (1) o documento norteador para implementação dos planos cicloviários nos municípios brasileiros, conhecido como "Caderno de Referência para elaboração do Plano de Mobilidade por Bicicleta nas Cidades" ou CBB (MINISTÉRIO DAS CIDADES, 2007b); (2) os critérios usados na elaboração do relatório "Política de Mobilidade por Bicicletas e Rede Cicloviária da Cidade de São Paulo: Análise e recomendações" do instituto de políticas de transporte e desenvolvimento (ITDP, 2015), do Brasil e (3) o índice "The Copenhagenize Index" (COPENHAGENIZE, 2018), por ser um recurso comumente aceito e utilizado para determinar a facilidade do trânsito das bicicletas nas cidades no mundo. 
O Que diz a Legislação sobre Critérios de Avaliação das Políticas e Ações de Mobilidade Cicloviária nos Municípios

Os primeiros passos em favor do planejamento cicloviário foram dados efetivamente na década de 1970, em março de 1976 a Empresa Brasileira de Planejamento de Transporte (GEIPOT, 2001), publicou a primeira edição do manual "Planejamento Cicloviário - Uma Política para as Bicicletas" após visita dos técnicos a Curitiba (PR) e Joinville (SC) para conhecer as iniciativas que estavam em andamento. Curitiba implantava sua rede cicloviária no interior de alguns parques e em conexões entre eles. Joinville, conhecida como a cidade com maior uso da bicicleta no Brasil.

Em maio de 2001, o Geipot elabora estudo que permitiu a atualização do Manual Cicloviário, e acredita que este estudo contribuiu significativamente para o processo de implantação de políticas cicloviárias no Brasil, pois dispõe de soluções adequadas a realidade nacional, como tipologias das pistas construídas e adaptadas para o país, revelando grande criatividade dos técnicos locais na solução de problemas e no atendimento da demanda dos ciclistas. Essas soluções foram desenvolvidas tanto para a geometria, como para a sinalização e para o pavimento das pistas implantadas (GEIPOT, 2001).

Em apoio à elaboração das políticas urbanas brasileiras, foi criado o Ministério das Cidades, em janeiro de 2003, e junto dela a Secretaria Nacional de Mobilidade Urbana (SeMob), que conta com um programa de ações para diversos projetos associados à mobilidade urbana, incluindo capacitação de equipes, investimentos diretos de recursos do orçamento da União e linhas de financiamento (MINISTÉRIO DAS CIDADES, 2007a).

Com o propósito de atribuir um caráter democrático e participativo, a obrigatoriedade conferida pela Lei Federal $n^{\circ} 10.257$ denominada Estatuto da Cidade, para o desenvolvimento da Política Nacional de Desenvolvimento Urbano - PNUD, em 2004 foi criado a Conferência Nacional das Cidades, com o objetivo de eleger e instalar o Conselho das Cidades (ConCidades) órgão colegiado de natureza deliberativa e consultiva, integrante da estrutura do Ministério das Cidades (MINISTÉRIO DAS CIDADES, 2004).

Na Resolução n 34, de 01 de julho de 2005 (BRASIL, 2005), o ConCidades emitiu orientações e recomendações sobre a aplicação do Estatuto da Cidade e dos demais atos normativos relacionados ao desenvolvimento urbano. Nele, também alterou o nome do Plano de Transporte Urbano Integrado para Plano Diretor de Transporte e da Mobilidade. A inclusão da palavra "mobilidade" teve o propósito de dar uma conotação mais voltada à qualidade do espaço urbano, justiça social, acessibilidade e mobilidade para as pessoas, em contraposição à cultura nacional do rodoviarismo. 
O Ministério das Cidades (MC), por meio da SeMob, criou o "Caderno de Referência para Elaboração de Plano de Mobilidade Urbana”, em 2007 (MINISTÉRIO DAS CIDADES, 2007a). O Caderno é um direcionador para os municípios, seu propósito é estimular as políticas públicas de trânsito, transporte e acessibilidade, e explicitamente dar prioridade ao transporte coletivo, ao transporte não motorizado e a implementação do conceito de acessibilidade universal.

O Ministério das Cidades por meio da SeMob também criou, em 2007, "Caderno Bicicleta Brasil", um caderno de referência para elaboração do Plano de Mobilidade por Bicicleta nas Cidades (MINISTÉRIO DAS CIDADES, 2007b). O Caderno Bicicleta Brasil reúne os subsídios necessários para os municípios que têm intenção de implantar um plano cicloviário, integrado aos demais modos existentes, formando uma rede de transporte, e por sua vez o estímulo da população ao uso da bicicleta.

Em 2012, após 17 anos de tramitação no Congresso Nacional, e depois de 9 anos de criação do Ministério das Cidades, o Ministério estabeleceu as diretrizes para a Política Nacional de Mobilidade Urbana (PNMU) através da Lei $n^{\circ}$ 12.587/2012, a chamada Lei de Mobilidade Urbana. A PNMU está fundamentada nos princípios de desenvolvimento sustentável das cidades, nas dimensões socioeconômicas e ambientais e foi instituída com objetivo de atender os artigos
182 e 183 da Constituição Federal de 1988, os quais atribuem à União a responsabilidade de criar diretrizes para a política de desenvolvimento urbano e de transportes, orientar a regulamentação e o planejamento da mobilidade urbana nas cidades brasileiras (BRASIL, 2012).

Foi a primeira vez que uma lei federal tratou diretamente da mobilidade urbana e incluiu o transporte não motorizado - a bicicleta -, o que denota o reconhecimento do transporte cicloviário (não motorizado). Assim a PNMU incorpora o conceito de mobilidade urbana sustentável, já presente na legislação de outros países. Dessa forma caberá aos municípios adotarem um novo conceito de planejamento de mobilidade urbana que priorize as pessoas, o modo de transporte cicloviário e os serviços de transporte público, em um planejamento de crescimento urbano de forma ordenada.

Logo no seu primeiro artigo, a PNMU já aborda a integração entre os modos de transporte motorizado com o não motorizado (bicicleta), bem como a acessibilidade e mobilidade urbana de pessoas e cargas no território do município. Determina, no Art. 24, que as cidades com mais de 20.000 (vinte mil) habitantes elaborem o Plano Diretor de Transporte e da Mobilidade integrado ao Plano Diretor Municipal, em um prazo de 3 (três) anos a contar da vigência da PNMU. Vale destacar que o prazo de 3 (três) anos estabelecido encerrou-se em abril de 2015 , tendo sido prorrogado por 
mais 3 (três) anos até abril de 2018 e novamente prorrogado por 1 (um) ano, até abril de 2019, data que marca o início da obrigatoriedade dos Planos Diretores de Transporte e Mobilidade integrados ao Planos Diretores Municipais como requisito para contratação de novas operações que utilizem recursos orçamentários federais (BRASIL, 2012). A baixa adesão por parte dos municípios aos planos acima referenciados demonstra a resistência dos municípios em lidar com o tema, e traz como consequência uma dificuldade de planejar e implementar políticas integradas de mobilidade urbana. Em virtude deste quadro, o tema avaliação destas políticas, torna-se algo distante da realidade da grande parte das cidades brasileiras, todavia imprescindíveis de serem estudadas pela própria obrigatoriedade legal dos planos de mobilidade.

Questões relativas à ciclomobilidade, que a princípio parecem apresentar ações isoladas ou de algum segmento da sociedade, hoje na realidade são direitos negados, haja visto que a partir de setembro de 2015 a Emenda Constitucional $n^{\circ}$ 90/2015 altera o texto do artigo $6^{\circ}$ da Constituição Federal em que o transporte passa a ser um direito social, ou seja, faz parte dos direitos fundamentais do cidadão tal como: lazer, alimentação, segurança, saúde, previdência social, educação, moradia, trabalho, proteção à maternidade e à infância e assistência aos desamparados (BRASIL, 2015). Esse direito passa a ter mais representatividade e impacto na mobilidade não motorizada quando a Pesquisa Nacional por Amostra de Domicílios (PNAD), realizada pelo Instituto Brasileiro de Geografia e Estatística (IBGE), destaca que mais de um terço, em torno de 30,6 milhões de trabalhadores no Brasil, em 2008, iam para o trabalho a pé ou de bicicleta, e quando a pesquisa era realizada entre os que residiam em domicílios cujo o rendimento domiciliar per capita era inferior a $1 / 4$ do salário mínimo, o percentual subia para $57,5 \%$ (IBGE, 2008).

Dado este breve histórico em que saímos dos primeiros passos com o Geipot focado na parte técnica de projetos de implantação cicloviária, chegamos a avanços do ponto de vista institucional e legal para uma política de desenvolvimento urbano que pensa a mobilidade, em especial a questão cicloviária de forma integrada. Mas do ponto de vista prático, esses avanços não se transformaram em mudanças significativas das cidades. A elaboração e implementação dos Planos de Mobilidade pelos municípios são um desafio, que com a obrigatoriedade legal, somadas a algumas experiências pioneiras na questão da avaliação e monitoramento cicloviário, passam a serem necessidades que devem ser atendidas, estudadas e aprimoradas. À medida que o prazo estabelecido para a elaboração dos Planos de Mobilidade se cumprir, os critérios de avaliação serão fundamentais para trazerem resultados em termos da evolução nas ações de implantação das políticas de mobilidade cicloviária. 
Uma Proposta de Critérios de Avaliação das Políticas Públicas Locais do Modal Cicloviário

Para demonstrar o universo geral dos critérios gerais de avaliação que se tem para questão cicloviária serão apresentados três referências, sendo:

O "Caderno de Referência para elaboração do Plano de Mobilidade por Bicicleta nas Cidades", conhecido como Caderno Bicicleta Brasil - CBB (MINISTÉRIO DAS CIDADES, 2007b), é o principal documento de orientação das políticas de mobilidade cicloviária do $\mathrm{MC}$, que na prática contribui significativamente com informações, pois abordam temas como a implantação do plano cicloviário básico para os municípios, elementos essenciais para planejamento cicloviário, projetos e integração da bicicleta com modos de transporte coletivo e estudos de caso de experiências com o modal cicloviário. Dessa forma pretendemos de maneira sistemática abordar os itens correspondentes aos valores originários do planejamento, projetos e experiências do CBB e aperfeiçoar como critérios para avaliar as políticas e ações de mobilidade cicloviária.

O ITDP (2015) em sua agenda de políticas públicas elabora o relatório "Política de Mobilidade por Bicicletas e Rede Cicloviária da Cidade de São Paulo: Análise e Recomendações" com o objetivo fornecer sugestões para o fortalecimento das políticas relacionadas à mobilidade por bicicletas, por meio da: identificação do histórico de ações, programas e políticas e análise in loco da rede cicloviária implantada no município de São Paulo de $359,9 \mathrm{~km}$ e do planejamento para implantação de mais $400 \mathrm{~km}$ de ciclovias na cidade de São Paulo. Os critérios de qualidade utilizados para avaliar as infraestruturas da rede cicloviária de São Paulo foram baseados, principalmente, em duas referências bibliográficas: Urban Bikeway Design Guide (NACTO, 2011) e Manual Ciclociudades (ITDP, 2011) do México que, por sua vez, traz referências do material holandês Sign up for the bike (CROW, 2011).

E por último uma experiência internacional, a do escritório Copenhagenize Design Company que publica índices sobre a taxa do uso da bicicleta, a qualidade da infraestrutura ciclista entre outros temas relacionados, haja vista que há mais de 100 anos a Dinamarca possui uma cultura ciclista que durante as últimas décadas proporcionou a Copenhagen algumas primeiras colocações no The Copenhagenize Index, que dá às cidades a classificação no ranking pelos seus esforços para reestabelecer a bicicleta como uma forma de transporte viável, aceita e prática (GAETE, 2015).

O ranking desde 2011 vem promovendo um desafio para todas as cidades do mundo com mais de 100.000 habitantes que tenham o interesse de levar o transporte cicloviário a sério, de maneira que os gestores venham a assumir às suas cidades, e entrar nesse desafio e se modernizar implemen- 
tando infraestrutura de bicicletas, políticas e ações em favor da ciclomobilidade. O ranking ocorre a cada dois anos e a classificação contempla as vinte primeiras cidades do mundo concedendo o reconhecimento pelos seus esforços para restabelecer a bicicleta como uma forma de transporte viável, aceita e prática. As cidades recebem entre zero e quatro pontos em quatorze parâmetros, para determinar a facilidade de utilizar a bicicleta em qualquer cidade, revelando o que está em vigor na cidade no momento da avaliação. O sistema de classificação foi desenvolvido em 2011, juntamente com James Schwartz, consultor de tecnologia, ciclista e autor de artigos relacionados à melhoria da infraestrutura cicloviária no site The Urban Country, fundado no ano de 2004, que tem como missão publicar artigos que apoiam e defendem o uso das bicicletas nas cidades. A inspiração foi obtida de classificações como o Monocle's Liveable Cities Index e os rankings produzidos pela The Economist (COPENHAGENIZE, 2018).

Esses documentos foram escolhidos por motivos variados. O CBB, por exemplo, é relevante porque agrega informações relativas à orientação legal para cidades brasileiras. Como experiência brasileira alinhada às políticas nacionais, através do relatório elaborado pelo ITDP São Paulo. Finalizamos esse levantamento com olhar para a experiência internacional em um documento de referência, como ranking reconhecido em diferentes países e cidades do mundo como o The Copenhagenize Index.
Com base nos documentos acima citados, primeiro apresentaremos cada uma dessas referências para identificação e compreensão dos critérios que consideraram relevantes na abordagem da temática; então, sistematizamos os principais critérios identificados; uma vez terminado o estudo dos três documentos, agrupamos os critérios em uma tabela e quantificamos sua repetição de forma a identificar os critérios que despontam como os mais recorrentes e, depreende-se daí, como os mais relevantes. Os critérios que aparecerem maior número de vezes serão considerados como os principais a serem elencados em uma proposta de referência como instrumento de avaliação para as políticas e ações públicas cicloviárias brasileiras.

\section{A orientação legal brasileira: Caderno Bicicle- ta Brasil}

O CBB considera cinco principais exigências a serem contempladas nos projetos e infraestrutura para as bicicletas e bem como algumas características e fatores que também são base para o plano cicloviário, e contribuem com informações fundamentais para dimensionar as políticas cicloviárias.

Dessa forma buscamos sistematizar os principais temas relacionados à avaliação da rede cicloviária e das políticas de mobilidade por bicicleta com base na leitura do CBB e apresentar conforme segue. 
As 5 principais exigências a serem contempladas nos projetos e infraestrutura para as bicicletas (MINISTÉRIO DAS CIDADES, 2007b):

Segurança viária - Envolve segurança viária dos ciclistas e dos demais usuários e aborda as seguintes medidas: projetos geométricos, medidas de moderação de tráfego, proteção física para pedestres e ciclistas, sinalização, fiscalização, tratamento das interseções, iluminação, piso;

Rotas diretas/rapidez/linearidade - Refere-se à redução do tempo de viagem e do esforço despendido pelo ciclista nos deslocamentos, sendo proporcionado por uma boa infraestrutura cicloviária que oferece rotas lineares e claras, sem desvios e interferências;

Coerência - A infraestrutura de uma rede cicloviária deve se apresentar de forma única (o desenho e dimensões devem compor visualmente uma unidade), contínua e coerente através de desenho facilmente reconhecível, manter a constância nas larguras das ciclovias e ciclofaixas e prover um sistema de informação e sinalização de rotas alternativas, trânsito e topografia;

Conforto - A infraestrutura cicloviária deve proporcionar um fluxo confortável, seguro e rápido para a circulação dos ciclistas, sendo abordado: escolha do piso, superfície regular, impermeável, antideslizante e de aspecto agradável. Além disso, é importante que as larguras sejam adequadas e que as rotas sejam protegidas do vento, chuva e sol;
Atratividade - A infraestrutura é desenhada de forma integrada ao meio ambiente em seu entorno, proporcionando um pedalar prazeroso, passando por ambientes atraentes e variados e com menor contato com as vias arteriais de trânsito rápido.

Com base nas exigências acima referenciadas o CBB elenca alguns fatores e características mais relevantes capazes de influenciar o uso ou não do modal cicloviário, e que também de igual forma contribuem para dimensionar as políticas cicloviárias (MINISTÉRIO DAS CIDADES, 2007b):

Qualidade física da infraestrutura - Esse fator considera a largura e o piso da via, a proteção lateral, os dispositivos sinalização para redução de velocidade na aproximação de cruzamentos e interseções perigosos e a iluminação;

Qualidade ambiental dos trajetos - preferencialmente trajetos integrados ao meio ambiente e evitar vias com veículos automotores com trânsito rápido;

Infraestrutura contínua - As vias cicláveis devem manter especialmente um nível de manutenção homogêneo de segurança de tráfego em todo o trajeto;

As intercessões - local que deve receber importância do tratamento diferenciado do restante das ciclovias por motivos de segurança; 
Facilidade para guardar a bicicleta - proporcionar ao ciclista a tranquilidade de guarda de sua bicicleta, dispor de estacionamentos seguros (bicicletários ou paraciclos) em vários pontos do espaço urbano e pontos de conexão entre modais;

Integração da bicicleta com outros modais este é um item essencial para o crescimento da mobilidade cicloviária e melhoria da mobilidade urbana. Nos locais de conexão deve existir espaço abrigado para a guarda segura das bicicletas, oficinas de apoio, banheiros, bebedouros de maneira que gerem atratividade pelo uso desses espaços e a permanência no uso do serviço de transporte público;

Informação e orientação - O trabalho de elaboração e divulgação de cartilhas e informativos para os ciclistas e outros segmentos da sociedade proporciona uma importante forma de orientação à construção de uma mentalidade cicloviária no meio urbano;

Capacitação Técnica - trata-se do fato de que os municípios devem buscar a capacitar seus técnicos junto ao próprio Ministério das Cidades, que tem programas específicos para prestar este auxílio às cidades;

Traffic Calming - Preparar a infraestrutura das cidades para a moderação do tráfego e redução da velocidade nas vias, proporcionando a diminuição de conflitos e acidentes com ciclistas.

\section{A experiência brasileira: o relatório do ITDP} sobre São Paulo

O relatório da Política de Mobilidade por Bicicletas e Rede Cicloviária da Cidade de São Paulo: Análise e Recomendações, afirma que a avaliação e monitoramento das políticas públicas cicloviárias são fundamentais para direcionar ações e recursos a serem aplicados. Dessa forma buscamos sistematizar os principais temas relacionados à avaliação da rede cicloviária e de políticas de mobilidade por bicicleta com base na leitura do relatório conforme segue (ITDP, 2015):

Segurança - Relacionado ao desenho viário deve proporcionar segurança aos usuários da via, sendo abordada a redução das velocidades praticadas (traffic calm), tratamento de interseções (sinalização horizontal, vertical, semaforização e elementos com desenhos geométricos), tratamento de interrupções com sinalização (Pontos de parada de ônibus e Escolas), separação dos tipos de veículos no caso de impossibilidade de compartilhamento viário;

Linearidade - Trata-se de uma rede cicloviária desenhada livre de obstáculos físicos, com possibilidade de atalhos seguros, continuidade dos percursos, que evitem mudanças de lado de circulação, sincronia dos semáforos e redução do número de intercessões; 
Coerência - Deve ser obtida através de uma coerência dos materiais de infraestrutura com linguagem uniforme (cores, formato, placas, sentidos de circulação, segregadores padronizados) Hierarquização viária facilmente reconhecível (vias de mesmo tipo devem possuir a mesma tipologia cicloviária), características físicas uniformes (larguras constantes, piso uniforme), vinculação com as linhas de desejo e abranger o máximo possível da área urbana de forma integrada permitindo fácil acesso das pessoas;

Conforto - Para tornar o deslocamento de bicicleta mais agradável a infraestrutura e equipamentos devem ter características físicas adequadas (largura, pavimentação, guias rebaixadas, rampas, travessias elevadas), Wayfinding (caminho encontrado) que corresponde a um conjunto de sistemas de informações na escala do ciclista; Atratividade - Proporcionada com integração de infraestruturas cicloviárias ao entorno e também estabelecê-la em locais atrativos. Alta densidade de destinos com polos geradores de viagens, praças, centros culturais, e outros equipamentos interessantes ligados diretamente ao percurso dos ciclistas como a instalação de paraciclos e bicicletários integrados ao transporte público (intermodalidade), sistema de bicicletas compartiIhadas e por último a segurança pública com vias e rotas obedecendo alguns requisitos como baixa velocidade, fácil acesso, iluminação, uso misto e fachadas ativas;
Extensão da Rede Cicloviária - Registro da extensão da rede cicloviária com a especificação em separado da extensão das vias dotadas de infraestrutura (ciclovias, ciclofaixas e ciclorrotas), independente de serem uni ou bidirecionais;

Divisão modal - Aponta o mapeamento dos locais com maior uso de bicicletas e o número total estimado de viagens realizadas em bicicletas e em outros modos de transporte;

Colisões envolvendo bicicletas e mortes de ciclistas - Registro das ocorrências fatais envolvendo ciclistas especializado no território e relacionar com a divisão/tipo de modal envolvido;

Tendências de uso da bicicleta - Contagens de ciclistas em termos quantitativo (volume de ciclistas) e qualitativo (gênero, faixa etária, dentre outros) com o propósito de identificar tendências (volumétricas e/ou qualitativas) do uso da bicicleta em regiões específicas ou distribuída pela cidade;

Cobertura e continuidade da rede - Mapeamento do percentual viário da rede com infraestrutura cicloviária contínua e eliminando a parte da rede fragmentada;

Qualidade da rede cicloviária - Pesquisas de satisfação e hábitos dos usuários; 
Alocação de recursos orçamentários - Trata-se de indicador referente ao orçamento, para monitorar as fontes e destino dos recursos, bem como monitorar os investimentos em infraestrutura cicloviária em comparação com a infraestrutura para outros modos de transporte e indicar a evolução anual.

\section{The Copenhagenize Index: a classificação no ranking mundial}

Para determinar a classificação no ranking mundial das cidades interessadas em ações em favor da ciclomobilidade, buscamos sistematizar os principais parâmetros utilizados com base na leitura do The Copenhagenize Index e apresentar conforme segue (COPENHAGENIZE, 2018):

Apoio Organizado - Refere-se à existência de algum apoio organizado ao modal cicloviário na cidade, como ONG's, grupos de cicloativistas e o grau de influência política que exercem;

Cultura de Bicicleta - Refere-se ao fato da bicicleta estar presente na paisagem urbana, como meio de transporte entre cidadãos comuns ou apenas em alguns grupos;

Instalações para Bicicletas - Se há bicicletários de fácil acesso, rampas para bicicletas nas escadas, espaço alocado em trens e ônibus para o transporte das bicicletas e orientações com sinalização de rotas ou caminhos para viagens planejadas;
Infraestrutura para Bicicletas - Refere-se como está à taxa de infraestrutura cicloviária (ciclovias, ciclofaixas e ciclorrotas) na cidade;

Programa de Compartilhamento de Bicicletas - A cidade possui um programa de compartilhamento de bicicletas abrangente no território urbano e é bem utilizado;

Divisão de Gênero - Refere-se qual o percentual de ciclistas da cidade é do sexo masculino e feminino;

Compartilhamento de modais com as bicicletas - Qual a porcentagem de participação modal cicloviário no compartilhamento de modais;

Aumento de participação de modal cicloviário - Trata-se de qual tem sido o percentual de aumento da participação modal cicloviário na cidade medido anualmente;

Percepção de Segurança - Trata-se da percepção de segurança dos ciclistas na cidade, é refletida através do aumento do uso de capacete, sendo negativa quando os ciclistas estão assustados devido às campanhas de promoção ou leis para o uso do capacete;

Política - Refere-se ao tratamento e a importância que a bicicleta tem na política e na gestão da cidade. Quanto mais envolvimento político e intenso da gestão, melhor a avaliação; 
Aceitação Social - Refere-se ao fato dos motoristas e a comunidade em geral consideram os ciclistas no meio urbano. É avaliado se não há nenhuma aceitação social ou se há uma aceitação social generalizada dos ciclistas na cidade;

Planejamento Urbano - Trata-se da ênfase que os planejadores dão cidade quanto à infraestrutura de bicicletas, se estão centrados em carros ou que pensam primeiro em bicicleta e em pedestres, e se os planejadores estão bem informados sobre as melhores práticas internacionais:

Traffic Calming (moderação de tráfego) - Trata dos esforços que foram feitos para diminuir os limites de velocidade - por exemplo, zonas de 30 $\mathrm{km} / \mathrm{h}$ - e, em geral, tranquilizar o tráfego, a fim de proporcionar maior segurança aos pedestres e ciclistas. Sendo avaliado a partir de nenhum nível de medidas de moderação de tráfego para medidas extremas de tráfego calmo, priorizando os ciclistas e pedestres na hierarquia viária;

Bicicletas de Carga e Logística - Mostra como as bicicletas de carga tem proporcionado uma alternativa crescente para mobilidade nas cidades. Dessa forma é avaliado se a cidade está abraçando o potencial das bicicletas de carga - tanto para os cidadãos quanto para as empresas.

Como resultado da investigação e da sistematização dos principais documentos de referência escolhidos, identificamos um total de 44 critérios abordados na avaliação do modal cicloviário. Esses critérios foram consolidados em uma única tabela (Figura 1) e identificamos aqueles que se repetem nos diferentes documentos. Quantificamos cada critério na coluna da direita, de título "Resultado".

Observa-se na coluna "resultado" que os critérios apareceram entre 1 e 3 vezes. Como critério de seleção e exclusão de alguns destes critérios, consideramos apenas os critérios que mais se repetiram, ou seja, se apareceram 2 ou 3 vezes nos documentos. A tabela com os principais critérios de avaliação de ciclomobilidade (Figura 2) é um recorte da tabela síntese dos critérios de avaliação de ciclomobilidade (Figura 1), e apresenta apenas os critérios selecionados. Como produto da síntese dos critérios de maior relevância, obtivemos como resultado um total de 18 critérios.

A proposta se organiza em Itens, que se referem aos principais aspectos considerados nas políticas de mobilidade cicloviária. Esses Itens são subdivididos em Critérios, que são as questões pontuais que $\mathrm{o}$ avaliador deverá levar em conta. Apresentamos esses Itens e Critérios na Figura 2 
Wellington Luiz de Azevedo, Flavia Nico Vasconcelos e Giovanilton André Carreta Ferreira Politicas de Mobilidade Urbana: Identificação de Critérios de Avaliação das Políticas e Ações de Ciclomobilidade

\begin{tabular}{|c|c|c|c|c|c|c|}
\hline \multicolumn{7}{|c|}{ SÍNTESE DOS CRITÉRIOS DE AVALIAÇÃO DE CICLOMOBILIDADE } \\
\hline$\underset{\mathrm{E}}{\stackrel{\sum}{E}}$ & 立 & CRITÉRIOS PARA AVALIAÇÃO DE CICLOMOBILIDADE: & $\begin{array}{c}\text { CADERNO } \\
\text { BICICLETA } \\
\text { BRASIL }\end{array}$ & \begin{tabular}{|l} 
RELÁTORIO \\
ITDP (2015) \\
SÃo PAULO
\end{tabular} & $\begin{array}{c}\text { RANKING } \\
\text { COPENHAGENIZE }\end{array}$ & 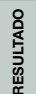 \\
\hline \multirow{14}{*}{ 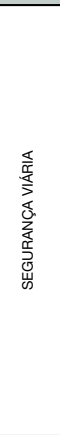 } & 1 & USO DO PROJETO GEOMÉTRICO NAS CICLOVIAS E SINALLZAÇÃO & $\mathrm{x}$ & $\mathrm{x}$ & & 2 \\
\hline & 2 & MODERAÇÃO DO TRÁFEGO (TRAFFIC CALMING) & $\mathrm{x}$ & $\mathrm{x}$ & $\mathrm{x}$ & 3 \\
\hline & 3 & $\begin{array}{l}\text { VISIBILIDADE ENTRE O CICLISTA E O CONDUTOR DE VEICULL, ELIMINANDO AS BARREIRAS } \\
\text { FiSICAS DESNECESSARRAS }\end{array}$ & $\mathrm{x}$ & & & 1 \\
\hline & 4 & $\begin{array}{l}\text { BARREIRAS DE PROTEÇÃO FíSICA PARA CICLISTAS COMO CANTEIROS, TACHŌES, GUIAS } \\
\text { EOUTROS }\end{array}$ & $\mathrm{x}$ & & & 1 \\
\hline & 5 & SIINALIZAC̣Ão HORIZONTAL, VERTICAL, SEMAFORIZAÇ̄̄O PADRONIZADAS & $x$ & $\mathrm{x}$ & & 2 \\
\hline & 6 & TRATAMENTO E REDUÇĀ̃O DO NÚMERO DE INTERCESSŌES & $x$ & $x$ & & 2 \\
\hline & 7 & ILUMINAÇĀO DAS CICLOVIAS & $x$ & $\mathrm{x}$ & & 2 \\
\hline & 8 & PISO/PAVIMENTO EM BOAS CONDIC̄ŌES & $x$ & & & 1 \\
\hline & 9 & TRATAMENTO DAS INTERRUPÇÖES & & $\mathrm{x}$ & & 1 \\
\hline & 10 & SEPARAÇÃO DOS TIPOS DE VEICULO & & $\mathrm{x}$ & & 1 \\
\hline & 11 & BICICLETÁRIOS E PARACICLOS & $x$ & $\mathrm{x}$ & $x$ & 3 \\
\hline & 12 & COLSŌES ENVOLVENDO BICICLETAS E MORTE DE CICLLSTAS & & $\mathrm{x}$ & & 1 \\
\hline & 13 & PERCEPCĀ̃o DE SEGURANÇA (USO DE CAPACETES) & & & $\mathrm{x}$ & 1 \\
\hline & 14 & QUALIDADE DA REDE CICLOVIÍARIA (SATISFAÇĀOO DO CICLISTA) & & $\mathrm{x}$ & & 1 \\
\hline \multirow{2}{*}{ 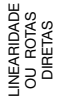 } & 15 & CONTINUIDADE DOS PERCURSOS, ROTAS LINEARES LIVRES DE OBSTACULOS FISIICOS & $\mathrm{x}$ & $\mathrm{x}$ & & 2 \\
\hline & 16 & EXTENSĀo dA REDE CICLOVIÍRIAA & & $\mathrm{x}$ & $\mathrm{x}$ & 2 \\
\hline \multirow{6}{*}{ 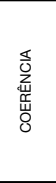 } & 17 & HIRARQUUZAC̣Āo VIÁRIA FACLLMENTE RECONHECIVEL & & $x$ & & 1 \\
\hline & 18 & $\begin{array}{l}\text { SINALIZACSÃ INFORMATIVA DAS ROTAS ALTERNATIVAS, TRÂNSITO, TOPOGRAFIA E } \\
\text { OUTROS }\end{array}$ & $\mathrm{x}$ & $\mathrm{x}$ & & 2 \\
\hline & 19 & CONSTÂNCIA NAS LARGURAS DAS CICLOVIAS E CICLOFAIXAS & $\mathrm{x}$ & $\mathrm{x}$ & & 2 \\
\hline & 20 & | INFRAESTRUTURA CONTINUA E COERENTE COM DESENHO FACLLMENTE RECONHECIDO & $x$ & $\mathrm{x}$ & & 2 \\
\hline & 21 & LINGUAGEM UNIFORME E PADRONIZADA (MATERIAIS E DIMENSŌES) & & $\mathrm{x}$ & & 1 \\
\hline & 22 & VINCULAÇĀO COM AS LINHAS DE DESEJO & & $\mathrm{x}$ & & 1 \\
\hline \multirow{3}{*}{ 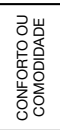 } & 23 & CARACTERRISTICAS FisICAS ADEQUADAS (INFRAESTRUTURA) - FLUXO CONFORTÁVEL & $\mathrm{x}$ & $\mathrm{x}$ & $\mathrm{x}$ & 3 \\
\hline & 24 & ROTAS PROTEGIDAS DO VENTO, SOL E CHUVA & $\mathrm{x}$ & & & 1 \\
\hline & 25 & WAYFINDING (CAMINHO ENCONTRADO) & & $\mathrm{x}$ & & 1 \\
\hline \multirow{15}{*}{ 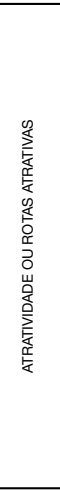 } & 26 & $\begin{array}{l}\text { INTEGRAÇ̄̃O DAS INFRAESTRUTURAS CICLOVIÍRIAS AO SEU ENTORNO E AO MEIO } \\
\text { AMBIENTE, BEM COMO LOCAIS ATRAENTES }\end{array}$ & $\mathrm{x}$ & $\mathrm{x}$ & & 2 \\
\hline & 27 & COINCIDIR O MiNIMO POSSIVELL COM AS VIAS ARTÉRIIAIS DE TRÂNSITO & $\mathrm{x}$ & & & 1 \\
\hline & 28 & $\begin{array}{l}\text { COINCIDIR COM LOCAIS DE ALTA DENSIDADE DE DESTINOS (PÓLOSS GERADORES DE } \\
\text { VIAGENS) }\end{array}$ & & $\mathrm{x}$ & & 1 \\
\hline & 29 & INTEGRAÇÃO COM OUTROS MODAIS & $\mathrm{x}$ & $\mathrm{x}$ & & 2 \\
\hline & 30 & ESPAÇOS ALOCADOS EM TRENS E OONIBUS PARA TRANSPORTE DAS BICICLETAS & & & $\mathrm{x}$ & 1 \\
\hline & 31 & 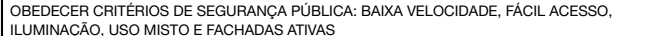 & & $\mathrm{x}$ & & 1 \\
\hline & 32 & AÇŌES DE EDUCAÇĀ̃O E PROMOC̣̄̃o DO USO DA BICICLETA & $x$ & & & 1 \\
\hline & 33 & CULTURA DA BICICLETA - A BICILLETA ESTA PRESENTE NA PAISAGEM URBANA & & & $\mathrm{x}$ & 1 \\
\hline & 34 & PROGRAMA DE COMPARTLLHAMENTO DE BICICLETAS & & $\mathrm{x}$ & $\mathrm{x}$ & 2 \\
\hline & 35 & $\begin{array}{l}\text { CIILLATIVISMO - APOIO DE ONG'S E ORGANIZAC̣ŌES CICLOATIVISTAS COM INFLLEENCIA } \\
\text { POLITICA }\end{array}$ & & & $\mathrm{x}$ & 1 \\
\hline & 36 & TENDÊNCIA DE USO DA BICICLLTTA COM A CONTAGEM DIVISĀO DE GËNERO E FAIXA ETÁRIIA & & $\mathrm{x}$ & $\mathrm{x}$ & 2 \\
\hline & 37 & DIVISÃO MODAL & & $\mathrm{x}$ & $\mathrm{x}$ & 2 \\
\hline & 38 & MAPEAMENTOS DOS LOCAIS COM MAIOR USO DE BICILLETAS & & $x$ & & 1 \\
\hline & 39 & PESQUISA SATISFAÇ̄̄O DA QUALIDADE DA REDE CICLOVVÍRIAA & & $\mathrm{x}$ & & 1 \\
\hline & 40 & ACEITAÇ̄̃O SOCIAL DOS CICLISTAS URBANOS & & & $\bar{x}$ & 1 \\
\hline \multirow{4}{*}{ 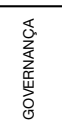 } & 41 & CAPACIDADE INSTITUCIONAL - POLITICA & & & $\mathrm{x}$ & 1 \\
\hline & 42 & ALOCAÇÃO DE INVESTIMENTOS & & $x$ & & 1 \\
\hline & 43 & PLANEJAMENTO CICLOVÍ́RIOO & $\mathrm{x}$ & & $\mathrm{x}$ & 2 \\
\hline & 44 & A CIDADE ESTÁ ABRAÇANDO O POTENCIAL DAS BICICLETAS DE CARGA & & & $\mathrm{x}$ & 1 \\
\hline
\end{tabular}

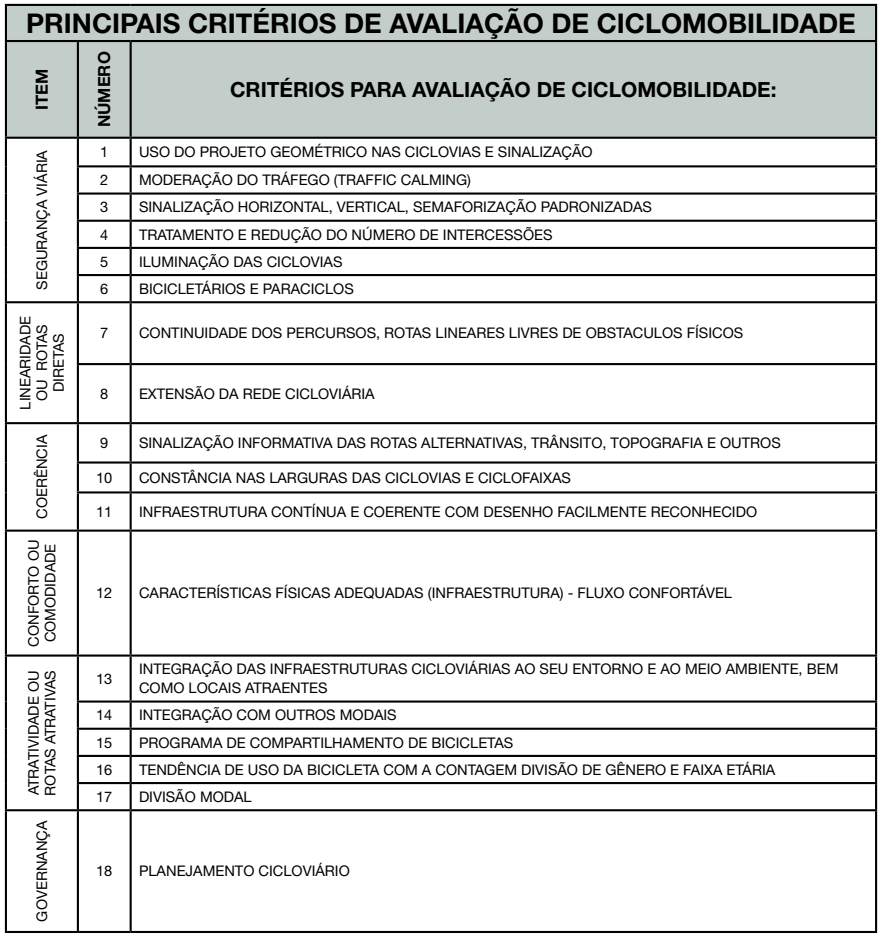

Figura 2. Instrumento de avaliação de política pública cicloviária nos municípios. Fonte: Desenvolvido pelo autor.

Figura 1. Síntese dos documentos de referência com resultado da relevância dos principais critérios de avaliação cicloviária. Fonte: Desenvolvido pelo autor.

usjt • arq.urb • número 25 | maio - agosto de 2019 


\section{Considerações Finais}

O padrão de mobilidade, centrado no transporte individual motorizado, trouxe a expectativa de resolver os problemas da mobilidade nas cidades, contudo as consequências negativas surgiram na mobilidade com a lentidão nos deslocamentos, com a poluição e piora na qualidade de vida das pessoas, tanto a nível local quanto ao impacto ambiental a nível global, revelando que a solução não está na exclusividade do automóvel no contexto da mobilidade.

O conceito ultrapassado de mobilidade tradicionalmente associado ao transporte, hoje está ampliado ao deslocamento de pessoas e cargas no espaço urbano priorizando o transporte coletivo e não motorizados relacionado, a qualidade de vida das pessoas e ao plano de desenvolvimento sustentável das cidades, e tem colaborado de forma significativa na promoção da bicicleta como um meio de transporte viável e capaz de interagir com outros modais.

De uma forma geral no cenário brasileiro as políticas públicas de mobilidade tem se apresentado favorável à implantação do modal cicloviário. Contudo na prática fica visível a falta de mecanismos de avaliação da implantação das políticas e ações concernentes ao modal cicloviário. A avaliação tem se mostrado uma ferramenta que proporciona aos gestores e a população condições de controle de medir o desenvolvimento e evolução das ações e políticas cicloviárias em seus municípios.
Diante dessa falta de mecanismos de avaliação, é oportuno utilizar documentos de referência que sirvam como instrumento norteador para elaborar os critérios de avaliação da implantação das políticas e ações cicloviárias, que sistematizados podem se converter em uma importante estratégia para o cumprimento das diretrizes para o Planejamento e Gestão dos Sistemas de Mobilidade Urbana e legislação, orientado no capítulo $\mathrm{V}$ da PNMU.

Podemos verificar que a sistematização de documentos de referência existente proporciona evidenciar os critérios mais relevantes por aparecerem mais vezes nos documentos estudados. E a partir desses critérios passamos a ter elementos para elaboramos uma proposta de instrumento de avaliação das políticas e ações de mobilidade cicloviárias, que proporciona a evolução e avanço nos municípios brasileiros em relação a mobilidade cicloviária.

Por fim, esse trabalho procurou evidenciar a necessidade de atentarmos para a orientação da PNMU quanto à implantação de mecanismos de avaliação das políticas e ações de mobilidade, bem como contribuir com a proposta de instrumento de avaliação das políticas e ações de mobilidade cicloviária nos municípios. 


\section{Referências:}

ANTP. ASSOCIAÇÃO NACIONAL DE TRANSPORTES PÚBLICOS. Transporte humano: Cidades com qualidade de vida. São Paulo, 1997.

BRASIL. MINISTÉRIO DAS CIDADES. Resolução $\mathbf{n}^{\circ} \mathbf{3 4}$, de $1^{\circ}$ de julho de 2005. Diário Oficial da União: Brasília, DF. 4 p. Disponível em: < https://www.cmc.pr.gov.br/down/pd_resolucao_federal_34-2005.pdf >. Acesso em: 13 ago. 2017.

BRASIL. MINISTÉRIO DAS CIDADES. Lei no 12.587, de 3 de janeiro de 2012. Institui as diretrizes da Política Nacional de Mobilidade Urbana (PNMU). Disponível em: < http://www.planalto. gov.br/ccivil_03/_ato2011-2014/2012/lei/l12587. htm>. Acesso em: 25 mar. 2018.

BRASIL. Constituição (1988). Emenda Constitucional $n^{\circ} \mathbf{9 0}$, de 15 de setembro de 2015. Dá nova redação ao art. $6^{\circ}$ da Constituição Federal, para introduzir o transporte como direito social. Disponível em: < http://www2.camara.leg.br/le$\mathrm{gin} / \mathrm{fed} / \mathrm{emecon} / 2015 /$ emendaconstitucional-90-15-setembro-2015-781520-publicacaooriginal-148098-pl.html>. Acesso em: 12 set. 2018.

CASLEY, Denis; KUMAR, Krishna. Project Monitoring and Evaluation in Agriculture. Washington, DC: World Bank, 1987.
COPENHAGENIZE. The Copenhagenize Index: Bicycle Friendly Cities Index 2017. 2018. Disponível em: < http://copenhagenizeindex.eu/01_ copenhagen.html\#>. Acesso em: 15 out. 2018.

CROW, Ede. Record 27: Manual de Diseño para el Tráfico de Bicicletas, 2011. < https:// pt.slideshare.net/ArchVicky/crow-record-27-manual-de-diseo-para-el-trfico-de-bicicletas-2011-Ir> Acesso em: 15 out. 2018.

GAETE, Constanza Martínez. As 20 cidades mais preparadas para o ciclismo urbano segundo o Ranking Copenhagenize 2015. Site de arquitetura Archdaily - on line, 17 de novembro de 2015. Disponível em: < https://www.archdaily. com.br/br/770915/as-20-cidades-mais-amigaveis-do-mundo-com-as-bicicletas-segundo-o-ranking-copenhagenize-2015>. Acesso em: 02 out. 2018.

GARCIA, Ronaldo Coutinho. Subsídios para organizar avaliações da ação governamental. Planejamento e Políticas Públicas, Brasília, 2001. Disponível em: < http://www.campinas.sp.gov. br/arquivos/recursos-humanos/txt_apoio_ronaldo_garcia.pdf>. Acesso em 15 out. 2018.

GEIPOT. Empresa Brasileira de Planejamento de Transportes. Planejamento Cicloviário: Diagnóstico Nacional. Brasília, 2001. 
IBGE. INSTITUTO BRASILEIRO DE GEOGRAFIA E ESTATÍSTICA. BRASIL. Pesquisa Nacional por Amostra nos Domicílios. 2008. Disponível em: < https://biblioteca.ibge.gov.br/visualizacao/monografias/GEBIS\%20-\%20RJ/panorama. pdf>. Acesso em: 12 mar. 2018.

IEMA. INSTITUDO DE ENERGIA E MEIO AMBIENTE. A bicicleta e as Cidades: Como inserir a bicicleta na política de mobilidade urbana. São Paulo, 2010. Disponível em: <https://iema-site-staging.s3.amazonaws.com/a_bicicleta_e_as_ cidades.pdf $>$. Acesso em: 29 out. 2018.

ITDP. INSTITUTO DE POLÍTICAS DE TRANSPORTE E DESENVOLVIMENTO. Política de Mobilidade por Bicicletas e Rede Cicloviária da Cidade de São Paulo: Análise e Recomendações. Brasil, São Paulo, novembro 2015. Disponível em:< http://itdpbrasil.org.br/wp-content/ uploads/2016/03/Relatorio-Clclovias-SP.pdf $>$. Acesso em: 28 jul. 2018.

ITDP. INSTITUTO DE POLÍTICAS PARA EL TRANSPORTE Y EL DESARROLLO. Manual Ciclociudades: Manual Integral de Movilidad Ciclista para Ciudades Mexicanas. Ciudad de México, junho 2011. Disponível em:<http://ciclociudades.mx/manual-ciclociudades/>. Acesso em: 28 jul. 2018.
MINISTÉRIO DAS CIDADES. Secretaria Nacional de Transportes e da Mobilidade Urbana. Política Nacional de Desenvolvimento Urbano - PNUD. Novembro 2004. Disponível em: < http://www. capacidades.gov.br/biblioteca/detalhar/id/103/ titulo/cadernos-mcidades-1--politica-nacional-de-desenvolvimento-urbano $>$. Acessado em: 02 abr. 2018.

Caderno PlanMob: Caderno de Referência para Elaboração de Plano de Mobilidade Urbana. Caderno para orientação aos órgãos gestores municipais na elaboração dos Planos Diretores de Mobilidade Urbana. Brasília: O Ministério, 2007a. Disponível em: < http://www.cidades.gov.br/images/stories/ArquivosSEMOB/ Biblioteca/LivroPlanoMobilidade.pdf>. Acesso em: 08 jun. 2017.

Programa Brasileiro de Mobilidade por Bicicleta: Caderno Bicicleta Brasil (CBB). Caderno de referência para elaboração de Plano de Mobilidade por Bicicleta nas Cidades. Brasília: Secretaria Nacional de Transporte e da Mobilidade Urbana, O Ministério 2007b. (Coleção Bicicleta Brasil). Disponível em: < http://www.intt.gob. ve/repositorio/biblioteca/texto_relacionados/ Livro_20Bicicleta_20Brasil.pdf>. Acesso em: 08 jun. 2017. 
Indicadores de efetividade da Política Nacional de Mobilidade Urbana. Relatório de atividades e resultados do grupo de trabalho para definição de indicadores para monitoramento e avaliação da efetividade da Política Nacional de Mobilidade Urbana. Brasília: Secretaria Nacional de Transporte e da Mobilidade Urbana (SNTMU), O Ministério, 2016. Disponível em: < http:// www.cidades.gov.br/images/stories/ArquivosSEMOB/publicacoes/relatorioindicadores 2018. pdf>. Acesso em: 08 jun. 2018.
NACTO, National Association of City Transportation Officials. Urban Bikeway Design Guide. 2011. Disponível em: <https://nacto. org/2011/03/11/nacto-launches-urban-bikeway-design-guide/ >. Acesso em: 28 jul. 2018. 